\title{
On the Worst-Case Analysis of Temporal-Difference Learning Algorithms
}

\author{
ROBERT E. SCHAPIRE \\ schapire@ research all.com \\ AT\&T Bell Laborateries, 600 Mountain Awente, Reom 24124 , Murray Hill, Ny 0707 . \\ MANFRED K. WARMIUTH \\ manfred@cse.ucsc.edu \\ Computer and Information Sciences, University of California, Santa Cruz, CA 95064
}

Editor: Leslie Pack Kaclbling

\begin{abstract}
We study the behavior of a family of leaming algorithrns based on Sutron's method ot temporal differences. In our on-line learning tramework learning takes place in a sequence of trials, and the goal of the learning algorithm is to estimate a discounted sum of all the remforcements that will be received in the fulure. In this setting, we are able to prove general upper bounds on the performance of a slightly modified version of Sutton's so-called TD $(\lambda)$ algorithm. These bounds are stated in terms of the performance of the best linear predictor on the given training sequence, and are proved without making any statisticat assumptions of any kind about the process producing the learner's observed training sequence. We also prove lower bounds on the performance of any algorithm for this learning problem and give a similar anatysis of the closely related problem of learning to predict in a model in which the leamer must produce predictions for a whole batch of observations before receiving reinforcement.
\end{abstract}

Keywords: machine Jearning, temporal-dirference learning, on-Jine tearning, worst-case analysis:

\section{Introduction}

As an example, consider the problem of estimating the present value of a company. At the end of each quarter $t$, a company returns a profit $\%$. In terms of its future profits. what is the company worth today? One possible answer is simply the sum lotal of all future profits $\sum_{k=0}^{\infty} r_{t+k}$, but this is elearly an unsatislactory measure of present worth since a dollar carned today is certainly worth more than a dollar earned ten years from now. Indecd, taking into account irflation and the exponential growth rate of money that is invested, it can be argued that future profits drop in value cxponentially with time.

For this reason, it is common to discount profits $r_{++}$earned $k$ time steps in the future by $\gamma^{k}$ : where $r<1$ is a parameter that estimates the rate at which future profits diminish in value. This leads to a definition of the present value of the company as the discounted sum

$$
y_{\ell}:-\sum_{k=0}^{x} \gamma^{k} r_{l-k}
$$

Suppose now that we want to predict or estimate the present value $t h$ as defined in Hiq. (1). Obviously, if we know all the future profits $r_{1}, r_{k, 1}, \ldots$, then we can compute $y_{t}$ directly, but it would be absurd to assume that the future is known in the present. 
Instead, we consider the problem of estimating $y_{t}$ based on current observations that can be made about the world and the company. We summarize these observations abstractly by a vector $\mathbf{x}_{t} \in \mathbb{R}^{N}$. This vector might include, for instance, the company's profits in recent quarters, current sales figures, the state of the economy as measured by gross national product, etc.

Thus, at the beginning of each quarter $t$, the veetor $\mathbf{x}_{t}$ is obscrved and an cstimatc $\hat{y}_{t} \in \mathbb{R}$ is formulated of the company's present value $y_{t}$. At the end of the quarter, the company returns profit $r_{t}$. The goal is to make the estimates $\hat{y}_{t}$ as close as possible to $y_{i}$.

We sludy this prediction problem more abstractly as follows: At each point in time $t$-.. $1,2, \ldots$, a learning agent makes an observation about the current state of its environment, which is summarized by a real vector $x_{t} \in 1 N^{N}$. After having made this observation, the learning agent receives some kind of feedback from its environment, which is summarized by a real number $r_{t}$. The goal of the learning agent is to learn to predict the discounted sum $y_{t}$ given in Eq. (1) where $\gamma \subset[0,1)$ is some fixed constant callod the discount rate parameter.

At each time step $t$, after receiving the instance vector $x_{t}$ and prior to receiving the reinforcement signal $r_{t}$, we ask that the learning algorithm make a prediction $\hat{y}_{t}$ of the value of $y_{t}$. We measure the performance of the lcarning algorithm in terms of the discrepancy between $\hat{y}_{t}$ and $y_{t}$. There are many ways of measuring this discrepancy; in this paper, we use the quadratic loss function. That is, we define the loss of the learning algorithm at time $t$ to be $\left(\hat{y}_{t}-y_{t}\right)^{2}$, and the loss for an entire sequence of predictions is just the sum of the losses at each trial. Thus, the goal of the learning algorithm is to minimize its loss over a sequence of observation/lecdback trials.

We study the worst-case behavior of a family of learning algorithms based on Sutton's (1988) method of temporal differences. Specifically, we analyze a slightly modificd version of Sutton's so called $\mathrm{TD}(\lambda)$ algorithm in a worst case framework that makes no statistical assumptions of any kind. All previous analyses of $\operatorname{TD}(\lambda)$ have relied heavily on stochastic assumptions about the nature of the environment that is generating the data observed by the learner (Dayan, 1992; Dayan \& Sejnowski, 1994; Jaakkola, Jordan \& Singh, 1993; Sutton, 1988; Watkins, 1989). For instance, the learner's environment is often modeled by a Markov process. We apply some of our results to Markov processes later in the paper.

The primary contribution of our paper is to introduce a method of worst-case analysis to the area of temporal-difference learning. We present upper bounds on the loss incurred by our temporal difference learning algorithm (denoted by $\operatorname{TD}^{*}(\lambda)$ ) which hold cren when the sequence of observations $\mathbf{x}_{t}$ and reinforcement signals $r_{t}$ is arbitrary.

To make our bounds meaningful in such an adversarial setting, we compare the performance of the learning algorithm to the loss that wotd be incurred by the best prediction function among a family of prediction functions: in this paper, this class will always be the set of linear prediction functions. More precisely, for any vector $\mathbf{u} \in \mathrm{H}^{N}$. let

$$
L^{\ell}(\mathbf{a}, S):-\sum_{t=1}^{\ell}\left(\mathbf{u} \cdot \mathbf{x}_{t}-y_{t}\right)^{2}
$$


denote the loss of vector $\mathbf{u}$ on the first $\ell$ trials of training sequence $S$. That is, $L^{\ell}(\mathbf{u}, S)$ is the loss that would be incurred by a prediction function that predicts $\mathbf{u} \cdot \mathbf{x}_{t}$ on each observation vector $\mathbf{x}_{\text {. }}$.

We compare the performance of our lcarning algorithms to the performance of the best vector $\mathbf{u}$ (of bounded norm) that minimizes the loss on the given sequence. For example, we prove below that, for any training sequence $S$, the loss on the first $\ell$ trials of $\operatorname{TD}^{*}(1)$ is at most $^{1}$

$$
\operatorname{minin}_{\substack{\|\mathbf{u}\| \leq U \\ L^{\ell}(\mathbf{u}, S) \leq K}}\left(L^{\ell}(\mathbf{u}, S)+2 \sqrt{K} U X_{0} c_{\gamma}+\|\mathbf{u}\|^{2} X_{0}^{2} c_{\gamma}^{2}\right)
$$

where $c_{\gamma}-(1+\gamma) /(1-\gamma)$. (Here, $U, X_{0}$ and $K$ are parameters that are used to "tune" the algorithm's "learning rate:" specifically, it is assumed that $\left\|\mathbf{x}_{t}\right\| \leq X_{0}$, and that $\min \left\{L^{\ell}(\mathbf{u}, S):\|\mathbf{u}\| \leq U\right\} \leq K$. Various methods are known for guessing these parameters when they are unknown; see, for instance, Cesa-Bianchi, Long and Warmuth's paper (1993).) Thus, $\operatorname{TD}^{*}(1)$ will perform reasonably well, provided that there cxists some linear predictor $\mathbf{u}$ that gives a good fit to the training sequence.

To better understand bounds such as those given in Eq. (2), it is often helpful to consider the average per-trial loss that is guaranteed by the bound. Suppose for the moment, as is likely to be the case in practice, that $U, X_{0}$ and $\gamma$ are fixed, and that $K$ grows lincarly with the number of trials $\ell$, so that $K-O(\ell)$. Then Eq. (2) implies that the average per-trial loss of $\mathrm{TD}^{*}(\mathrm{i})$ (i.e., the total cumulative loss of $\mathrm{TD}^{*}(1)$ divided by the number of trials $\ell$ ) is at most

$$
\min _{\substack{\|\mid \mathbf{u}\|<\ell \\ L^{\ell}(\mathbf{u}, S) \leq K}}\left(\frac{L^{\ell}(\mathbf{u}, S)}{\ell}+O\left(\frac{1}{\sqrt{\ell}}\right)\right) .
$$

In other words, as the number of trials $\ell$ becomes large, the average per-trial loss of $\mathrm{TD}^{*}(1)$ rapidly approaches the average loss of the best vector $\boldsymbol{u}$. Furthermore, the rate of convergence is given explicitly as $O(1 / \sqrt{\ell})$.

Note that the above result, like all the others presented in this paper, provides a characterization of the learner's performance after only a finite number of time steps. In contrast, most previous work on $\operatorname{TD}(\lambda)$ has focused on its asymptotic performance. Moreover, previous researchers have focused on the convergence of the learner's hypothesis to a "true" or "optimal" model of the world. We, on the other hand, take the view that the learner's one and only goal is to make good predictions, and we thereforc measure the learner's performance entirely by the quality of its predictions.

The upper bound given in Eq. (2) on the performance of $\mathrm{TD}^{*}(1)$ is derived from a more general result we prove on the worst-case performance of $\operatorname{TD}^{*}(\lambda)$ for general $\lambda$. Our bounds for the special case when $\lambda-0$ or $\lambda=1$ can be stated in closed form. The proof techniques used in this paper are similar but morc general than those used by Cesa-Bianchi, Long and Warmuth (1993) in their analysis of the Widrow-Hoff algorithm (corresponding to the case that $\gamma-0$ ). 
Note that $\min \left\{L^{\ell}(\mathbf{u}, S): \mathbf{u} \in \mathbb{R}^{N}\right\}$ is the best an arbitrary linear model can do that knows all $y_{1} \cdots y_{\ell}$ ahead of time. If the on-line learner were given $y_{t}$ at the end of trial $t$ (i.c., if $\gamma-0$ ), then the Widrow-Hoff algorithm would achieve a worst case bound of

$$
\begin{aligned}
& \min _{:|\mathbf{u}| \mid<0}\left(L^{\ell}(\mathbf{u}, S)+2 \sqrt{K} U X_{0}+\|\left.\mathbf{u}\right|^{2} X_{0}{ }^{2}\right) \\
& I^{2}(\mathrm{u} . S) \leq K
\end{aligned}
$$

(matching the bound in Eq. (2) with $\gamma$ set to 0 ). However, in our model, the learner is given only the reinforcements $r_{t}$, even though its goal is to accurately estimate the infinite sum $y_{i}$ given in Eq. (1). Intuitively, as $\gamma$ gets larger, this task becomes more difficult since the learner must make predictions about events farther and farther into the future. All of our worst-case loss bounds depend explicitly on $\gamma$ and, not surprisingly, these bounds typically tend to infinity or become vacuous as $\gamma$ approaches 1 . Thus, our bounds quantify the price one has to pay for giving the learner successively less information.

In addition to these upper bounds, we prove a general lower bound on the loss of any algorithm for this prediction problem. Such a lower bound may be helpful in determining what kind of worst-case bounds can feasibly be proved. None of our upper bounds match the lower bound; it is an open question whether this remaining gap can be closed (this is possible in certain special cases, such as when $\gamma=0$ ).

Finally, we consider a slightly different, but closely related learning model in which the learner is given a whole batch of instances at once and the task is to give a prediction for all instances before an outcome is received for each instance in the batch. The loss in a trial $t$ is $\left.\left|\hat{\mathbf{y}}_{t} \quad \mathbf{y}_{t}\right|\right|^{2}$, where $\hat{\mathbf{y}}_{t}$ is the vector of predictions and $\mathbf{y}_{t}$ the vector of outcomes. Again, the goal is to minimize the additional tocal loss summed over all trials in excess of the total loss of the best linear predictor (of bounded norm).

In this batch model all instances count equally and the exact outcome for each instance is received at the end of each batch. A special case of this model is when the algorithm has to make predictions on a whole batch of instances before receiving the same outcome for all of them (a case studied by Sutton (1988)).

We again prove worst-case bounds for this model (extending Cesa-Bianchi, Long and Warmuth's (1993) previous analysis for the noise free case). We also prove matching lower bounds for this very general model, thus proving that our upper bounds are the optimal worst-case bounds.

The paper is outlined as follows. Section 2 describes the on-line model for temporal difference learning. Section 3 gives Sutton's original temporal difference learning algorithm $\mathrm{TD}(\lambda)$ and introduces our new algorithm $\mathrm{TD}^{*}(\lambda)$. Section 4 contains the worst-case loss bounds for the new algorithm, followed by Section 5 containing a lower bound for the on-line model. In Section 6, we illustrate our results with an application of $\mathrm{TD}^{*}(1)$ to obtain a kind of convergence result in a Markov-process setting. We present our results for the batch model in Section 7. Finally, we discuss the merits of the method of worst-case analysis in Section 8 . 


\section{The prediction model}

In this section, we describe our on line learning model. Throughout the paper, $N$ denotes the dimension of the learning problem. Each trial $t(t=1,2, \ldots)$ proceeds as follows:

1. The learner receives instance vector $\mathbf{x}_{t} \subset \mathbb{R}^{N}$.

2. The learner is required to compute a prediction $\hat{y}_{t} \in \mathbb{R}$.

3. The learner receives a reinforcement signal $r_{t} \in \mathrm{K}$.

The goal of the tearner is to predict not merely the next reintorcement signal, but rather a discounted sum of all of the reinforcements that will be received in the future. Specifically, the learner is trying to make its prediction $\hat{y}_{t}$ as close as possible to

$$
y_{t}:=\sum_{k=0}^{\infty} \gamma^{k} \gamma_{t+k}
$$

where $\gamma \in[0,1$ ) is a fixed parameter of the problem. (We will always assume that this infinite suni converges absulutely for all $t$.)

Note that if we multiply $y_{t}$ by the constant $1-\gamma$, we obtain a weighted average of all the future $r_{t}$ 's; that is, $(1-\gamma) y_{t}$ is a weighted average of $r_{t}, r_{t+1}, \ldots$. Thus it might be more natural to use the variables $y_{t}^{\prime}=y_{t}(1-\gamma)$. (For instance, if all $r_{t}$ equal $r$, then the modified variables $y_{t}^{\prime}$ all equal $r$ as well.) However, for the sake of notational simplicity, we use the variables $y_{t}$ instead (as was done by Sutton (1988) and others).

The infinite sequence of pairs of instances $\mathbf{x}_{t}$ and remforcement signals $r_{t}$ is called a training sequence (usually denoted by $S$ ). The loss of the learner at trial $t$ is $\left(y_{t}-\hat{y}_{t}\right)^{2}$, and the total loss of an algorithm $A$ on the first $\ell$ trials is

$$
L^{\ell}(A, S):-\sum_{t=1}^{\ell}\left(y_{t} \cdots \cdots \hat{y}_{t}\right)^{2} .
$$

Similarly, the total loss of a weight vector $\mathbf{u} \in \mathbb{R}^{N}$ on the first $\ell$ trials is defined to be

$$
L^{\ell}(\mathbf{u}, S):=\sum_{t=1}^{\ell}\left(y_{t}-\mathbf{u} \cdot \mathbf{x}_{t}\right)^{2}
$$

The purpose of this paper is to exhibit algorithms whose loss is guaranteed to be "not too much worse" than the loss of the best weight vector for the entire sequence. Thus, we would like to show that if there exists a weight vector $\mathbf{u}$ that fits the training sequence well, then the learner's predictions will also be reasonably good 


\section{Temporal-difference algorithms}

Wo focus now on a family of lcarning algorithms that arc only a slight modification of those considered by Sutton (1988). Each of these algorithms is parameterixed by a real number $\lambda \Leftarrow 0,1]$. For any sequence $S$ and $t=1,2, \cdots$, let

$$
\mathbf{X}_{t}^{\lambda}:-\sum_{k=1}^{t}(\gamma \lambda)^{t-k} \mathbf{x}_{k}
$$

be a weighted sum of all previously observed instances $\mathbf{x}_{k}$. The parameter $\lambda$ controls how strong an influence past instances have. For instance, when $\lambda=0, \mathbf{X}_{t}^{0}=\mathbf{x}_{t}$ so only the most recent instance is considered.

The learning algorithm $T D(\lambda)$ works by maintaining a weight vector $\mathbf{w}_{t} \in \mathbb{R}^{N}$. The initial weight vector $\mathbf{w}_{\perp}$ may be arbitrary, although in the simplest case $\mathbf{w}_{1}-\mathbf{0}$. The werght vector $\mathbf{w}_{t}$ is then updated to the new weight vector $\mathbf{w}_{t+1}$ using the following update rule:

$$
\mathbf{w}_{t+1}:-\mathbf{w}_{\ell}+\eta_{t}\left(r_{t}+\gamma \hat{y}_{t+1} \cdots \hat{y}_{t}\right) \mathbf{x}_{t}^{\lambda} .
$$

As suggested by Sutton (1988), the weight vectors are updated using $\mathbf{X}_{t}^{\lambda}$ rather than $\mathbf{x}_{t}$, allowing instances prior to $\kappa_{t}$ to have a diminishing influcnec on the update.

The constant $\eta_{t}$ appearing in Eq. (4) is called the learning rate on trial $t$. We will discuss later how to set the learning rates using prior knowledge about the training sequence.

In Sutton's original presentation of $T D(\lambda)$, and in most of the subsequent work on the algorithm, the prediction at each step is simply $\hat{y}_{t}-\mathbf{w}_{t} \cdot \mathbf{x}_{t}$. We, however, have found that a variant on this prediction rule leads to a simpler analysis, and, moreover, we were unable to obtain worst-case loss bounds for the original algorithm $\mathrm{TD}(\lambda)$ as strong as the bounds we prove for the new algorithm.

Our variant of $\operatorname{TD}(\lambda)$ uses the same update (4) for the weight veetor as the original algorithm, but predicts as follows:

$$
\begin{gathered}
\hat{y}_{t} \quad \cdots-\mathbf{w}_{t} \cdot \mathbf{x}_{t}+\sum_{k: 1}^{t-1}(\gamma \lambda)^{t}{ }^{k}\left(\mathbf{w}_{t} \cdot \mathbf{x}_{k}-\hat{y}_{k}\right) \\
-\mathbf{w}_{t} \cdot \mathbf{X}_{t}^{\lambda}-\sum_{k=1}^{i \cdots 1}(\gamma \lambda)^{t-k} \hat{y}_{k} .
\end{gathered}
$$

This new algorithm, which we call $\operatorname{TD}^{*}(\lambda)$, is summarized in Fig. 1.

The rule (4) for updating $\mathbf{w}_{t+1}$ has $\mathbf{w}_{t+1}$ implicit in $\hat{y}_{t+1}$, so at first it scems impossible to do this update rule. ${ }^{2}$ However, by multiplying Eq. (4) by $\mathbf{X}_{l+1}^{\lambda}$, one can first solve for $\hat{y}_{t+1}$ and then compute $w_{t+1}$. Specifically, this gives a solution for $y_{t+1}$ of

$$
\frac{\left(\mathbf{w}_{t} \mid \eta_{t}\left(r_{t}-\hat{y}_{t}\right) \mathbf{X}_{t}^{\lambda}\right) \cdot \mathbf{X}_{t+1}^{\lambda} \cdots \sum_{k=1}^{t}(\gamma \lambda)^{t+1 \cdots k} \hat{y}_{k}}{1-\eta_{t} \mathbf{X}_{t}^{\lambda} \cdot \mathbf{X}_{t+1}^{\lambda}}
$$




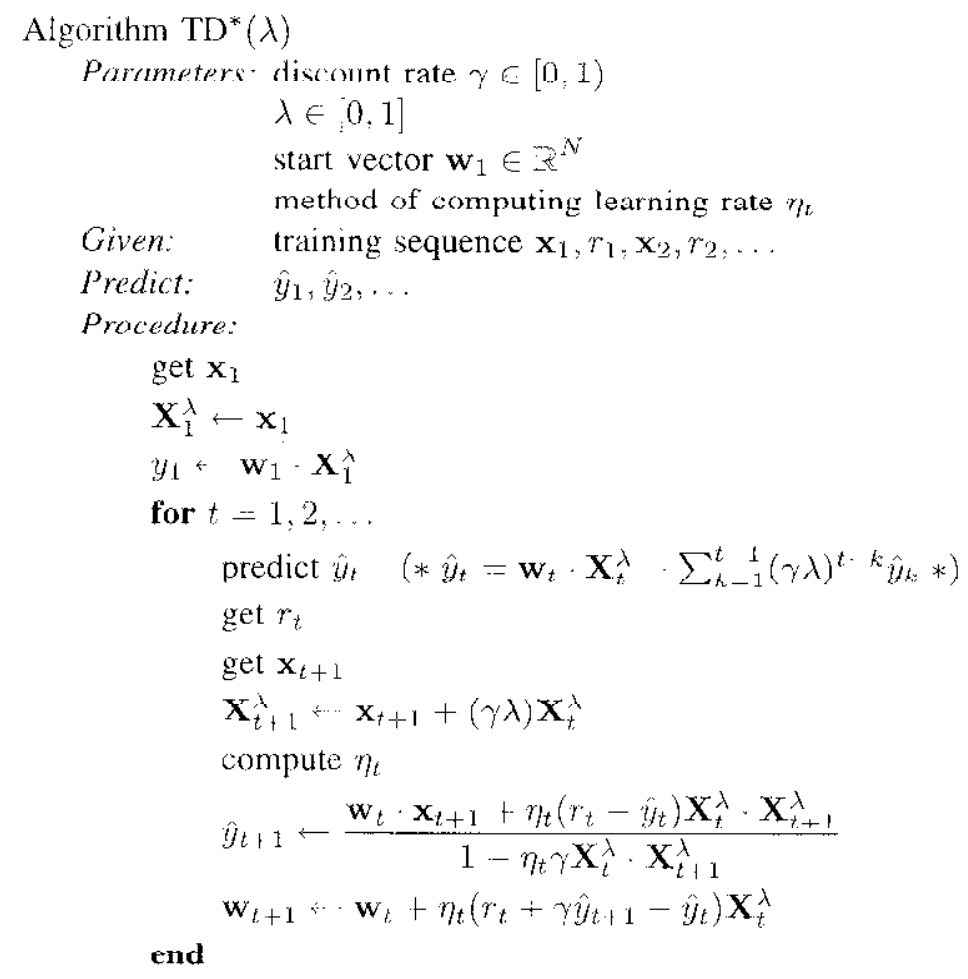

Figure 1. Pseudocode for $\mathrm{TD}^{*}(\lambda)$.

$$
\begin{aligned}
& =\frac{\left(\mathbf{w}_{t} \mid \eta_{t}\left(r_{t}-\hat{y}_{t}\right) \mathbf{X}_{t}^{\lambda}\right) \cdot \mathbf{X}_{t+1}^{\lambda}-(\gamma \lambda) \mathbf{w}_{t} \cdot \mathbf{X}_{t}^{\lambda}}{1-\eta_{t} \gamma \mathbf{X}_{t}^{\lambda} \cdot \mathbf{X}_{t+1}^{\lambda}} \\
& =\frac{\mathbf{w}_{t} \mathbf{x}_{t+1}: \eta_{t}\left(r_{t} \hat{y}_{t}\right) \mathbf{X}_{l}^{\lambda} \mathbf{X}_{t \mid 1}^{\lambda}}{1-\eta_{t} \gamma \mathbf{X}_{t}^{\lambda} \cdot \mathbf{X}_{l+1}^{\lambda}}
\end{aligned}
$$

where, in the first equality, we assume inductively that Fa. (5) holds at trial t. Thus, we can solve successfully for $\hat{y}_{t} .1$ provided that $\eta_{\eta} \mathbf{X}_{i}^{\lambda} \cdot \mathbf{X}_{i+1}^{\lambda}+1$, as will be the case for all the values of $\eta$. we consider. Also, note that $y+1$ is computed after the instance $\mathbf{x}_{i+1}$ is received but before the reinforcement $r_{t, 1}$ is available (see Fig. I for details).

Note that for the prediction $\hat{y}_{t}-\mathbf{w}_{i} \cdot \mathbf{x}_{t}$ of $\operatorname{TD}(\lambda)$,

$$
\begin{aligned}
\nabla_{\mathbf{w}_{i}}\left(y_{t} \quad y_{i}\right)^{2} & =-2 \eta_{t}\left(y_{t}-y_{t}\right) \mathbf{x}_{t} \\
& \approx-2 \eta_{t}\left(r_{t}-\gamma_{t} \cdot-\hat{y}_{t}\right) \mathbf{x}_{t} .
\end{aligned}
$$

(Since $y_{t} \cdots r_{i}+\gamma y_{t+1}$ is not available to the learner, it is approximated by $r_{l}+\gamma \hat{\eta}_{-1}$ ) Thus with the prediction rule of $\mathrm{TD}(\lambda)$ the update rule (4) is not gradient descent for 
all choices of $\lambda$. In contrast, with the new prediction rule (5) of $\mathrm{TD}^{*}(\lambda)$, the update rule (4) used by both algorithms is gradient descent, ${ }^{3}$ since if $\hat{y}_{t}$ is set according to the new prediction rule then

$$
\begin{aligned}
\nabla_{\mathbf{w}_{t}}\left(y_{t} \cdot \hat{y}_{t}\right)^{2} & =-2 \eta_{t}\left(y_{t}-\hat{y}_{t}\right) \mathbf{X}_{t}^{\lambda} \\
& \approx-9 \eta_{t}\left(r_{t}+\gamma \hat{y}_{t+1}-\hat{y}_{t}\right) \mathbf{X}_{t}^{\lambda}
\end{aligned}
$$

We can also motivate the term $-\sum_{k=1}^{t-1}(\gamma \lambda)^{t-k} \hat{y}_{k}$ in the prediction rule of $\operatorname{TD}^{*}(\lambda)$ given in Eq. (5): In this paper, we are comparing the total loss of the algorithm with the total loss of the best linear predictor, so both algorithms $\operatorname{TD}(\lambda)$ and $\operatorname{TD}^{*}(\lambda)$ try to match the $y_{t}$ 's with an on-line linear model. In particular, if $y_{t}=\mathbf{w} \cdot \mathbf{x}_{t}$ (that is, the $y_{t}$ 's are a linear function of the $\mathrm{x}_{i}$ 's) and the initial weight vector is the "correct" weight vector $\mathbf{w}$, then the algorithms should always predict correctly (so that $\hat{y}_{t}-y_{t}$ ) and the weight vector $\mathbf{w}_{l}$ of the algorithms should remain unchanged. It is easy to prove by induction that both algorithms have this property.

Thus, in sum, the prediction rule $\hat{y}_{t}=\mathbf{w}_{t} \cdot \mathbf{X}_{t}^{\lambda}+c_{t}$ is motivated by gradient descent, where $c_{t}$ is any term that does not depend on the weight vector $\mathbf{w}_{t}$. The exact value for $c_{t}$ is derived using the fact that, in the case described above, we want $\grave{y}_{t}=y_{t}$ for all $t$.

\section{Upper bounds for $\mathrm{TD}^{*}(\lambda)$}

In proving our upper bounds, we begin with a very gencral lemma concerning the performance of $\operatorname{TD}^{*}(\lambda)$. We then apply the lemma to derive an analysis of some special cases of interest.

LEMMA 1 let $\gamma \in[0,1), \lambda \in[0,1]$, and let $S$ be an arbitrary training sequence such that $\left\|\mathbf{X}_{t}^{\lambda}\right\| \leq X_{\lambda}$ for all trials $t$. Let $\mathbf{u}$ be any weight vector, and let $\ell>0$.

If we execute $\operatorname{TD}^{*}(\lambda)$ on $S$ with initial vector $\mathrm{w}_{1}$ and learning rates $\eta_{t}=\eta$ where $0<\eta X_{\lambda}{ }^{2} \gamma<1$, then

$$
L^{\ell}\left(\mathrm{TD}^{*}(\lambda), S\right)<\inf \left\{\frac{b L^{\ell}(\mathbf{u}, S)+\left\|\mathbf{u}-\mathbf{w}_{1}\right\|^{2}}{C_{b}}: b>0, C_{b}>0\right\}
$$

where $C_{b}$ pquots

$$
\begin{aligned}
& 2 \eta \cdot \eta^{2} X_{\lambda}^{2}\left(1-\gamma^{2}\right) \quad \frac{\eta^{2}}{b}\left(1+\left(\frac{\gamma \cdots \gamma \lambda}{1-\gamma \lambda}\right)^{2}\right) \\
& \quad \cdots 2\left(\left|\eta-\frac{\eta^{2}}{b}\right|\right)\left(\frac{\gamma-\gamma \lambda}{1-\gamma \lambda}\right) \gamma \lambda \cdots 2\left(!\left(\eta-\frac{\eta^{2}}{b}\right)(\gamma-\gamma \lambda)-\eta^{2} X_{\lambda}^{2} \gamma \mid\right) .
\end{aligned}
$$

Proof: For $1 \leq t \leq \ell$, we let $e_{t}-y_{t}-\hat{y}_{t}$, and $\epsilon_{\mathrm{u}, t}=y_{t} \cdots \mathbf{u} \cdot \mathbf{x}_{t}$. We further define $e_{\ell+1} \cdots 0$. Note that the loss of the algorithm at trial $t$ is $c_{t}{ }^{2}$ and the loss of $\mathbf{u}$ is $e_{\mathbf{1} 2} t^{2}$. Since, for $l<\ell$.

$$
\begin{aligned}
r_{t}+\gamma \hat{y}_{t+1}-y_{t} & -r_{t}+\gamma \hat{y}_{t+1} \quad\left(r_{t}+\gamma y_{t+1}\right)+y_{t}-y_{t} \\
& -e_{t} \cdots \gamma e_{t+1}:
\end{aligned}
$$


we can write $\mathrm{Eq}$. (4), the update of our algorithm, conveniently as

$$
\mathbf{w}_{t+1}=\mathbf{w}_{t}+\eta\left(e_{t}-\gamma e_{t+1}\right) \mathbf{X}_{t}^{\lambda} \text {. }
$$

To simplify the proof, we also define

$$
\mathbf{w}_{\ell+1}=\mathbf{w}_{\ell}+\eta_{\ell} \mathbf{X}_{\ell}^{\lambda}
$$

so that Eq. (6) holds for all $t \leq \ell$. (In fact, this definition of $\mathbf{w}_{\ell+1}$ differs from the vector that would actually be computed by $\operatorname{TD}^{*}(\lambda)$. This is not a problem, however, since we are here only interested in the behavior of the algorithm on the first $\ell$ trials which are unaffected by this change.)

We use the function progr $r_{+}$to measure how much "closer" the algorithm gets to $\mathbf{u}$ during trial $t$ as measured by the distance function $\|\cdot\|^{2}$ :

$$
\operatorname{progr}_{\ell}-\left\|\mathbf{u}-\mathbf{w}_{t}\right\|^{2}-\left\|\mathbf{u}-\mathbf{w}_{t+1}\right\|^{2} .
$$

Let $\Delta \mathbf{w}_{t}-\mathbf{w}_{t+1}-\mathbf{w}_{t}$ for $t \leq \ell$. We have that

$$
\begin{aligned}
\left\|\Delta \mathbf{w}_{t}\right\|^{2} & -\eta^{2}\left(e_{t}-\gamma e_{t+1}\right)^{2}\left\|\mathbf{X}_{t}^{\lambda}\right\|^{2} \\
& <\eta^{2} X_{\lambda}{ }^{2}\left(e_{t} \quad \gamma e_{t+1}\right)^{2}
\end{aligned}
$$

and that

$$
\begin{aligned}
\Delta \mathbf{w}_{t} \cdot\left(\mathbf{w}_{t}-\mathbf{u}\right) & =\eta\left(e_{t}-\gamma e_{t+1}\right)\left(\mathbf{w}_{t} \cdot \mathbf{X}_{t}^{\lambda}-\mathbf{u} \cdot \mathbf{X}_{t}^{\lambda}\right) \\
& =\eta\left(e_{t}-\gamma e_{t+1}\right) \sum_{k=1}^{t}(\gamma \lambda)^{t-k}\left(\hat{y}_{k}-\mathbf{u} \cdot \mathbf{x}_{k}\right) \\
& =\eta\left(e_{t}-\gamma e_{t, 1}\right) \sum_{k=1}^{t}(\gamma \lambda)^{t-k}\left(e_{\mathbf{u}, k_{t}}-e_{t_{t}}\right)
\end{aligned}
$$

where the second equality follows from Eqs. (3) and (5), and the last equality from the fact that

$$
\begin{gathered}
\hat{y}_{k} \quad \mathbf{u} \cdot \mathbf{x}_{k}-\hat{y}_{k} \quad y_{k}+y_{k}-\mathbf{u} \cdot \mathbf{x}_{k} \\
e_{\mathbf{u}, k}-e_{k} .
\end{gathered}
$$

Since $-\operatorname{progr}_{t}-2 \Delta \mathbf{w}_{t} \cdot\left(\mathbf{w}_{t}-\mathbf{u}\right)+\left.\Delta \mathbf{w}_{t}\right|^{2}$, we have that

$$
\begin{aligned}
& \begin{array}{ll}
\mathbf{w}_{1} \quad \mathbf{u} \|^{2} & \leq\left\|\mathbf{w}_{\ell+1} \quad \boldsymbol{u}\right\|^{2}
\end{array}\left|\begin{array}{ll}
\mid \mathbf{w}_{\perp} & \mathbf{u}
\end{array}\right|^{2} \\
& -\sum_{t=1}^{\ell} \operatorname{progr}_{t}
\end{aligned}
$$

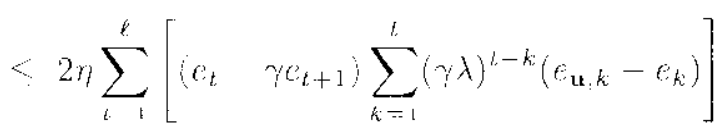

$$
\begin{aligned}
& +\eta^{2} X_{\lambda}^{2} \sum_{t=1}^{\ell}\left(e_{t}-e_{t+1}\right)^{2} \text {. }
\end{aligned}
$$


This can be written more concisely using matrix notation as follows: Let $\mathbf{Z}_{k}$ be the $\ell \times \ell$ matrix whose entry $(i, j)$ is defined to be 1 if $j=i+k$ and 0 otherwise. (For instance, $\mathbf{Z}_{0}$ is the identity matrix.) Let $\mathbf{D}-\mathbf{Z}_{0} \cdots \gamma \mathbf{Z}_{1}$, and let

$$
\mathbf{V}-\sum_{t: 0}^{\ell=1}(\gamma \lambda)^{t} \mathbf{Z}_{t}
$$

Finally, let $\mathbf{e}$ (respectively, $\mathbf{e}_{\mathrm{u}}$ ) be the length $\ell$ vector whose $t^{\text {th }}$ element is $e_{\ell}$ (respectively, $e_{\mathbf{u}, t}$ ). Then the last expression in Eq. (7) is equal to

$$
\eta^{2} X_{\lambda}{ }^{2} \mathbf{e}^{T} \mathbf{D}^{T} \mathbf{D e}+2 \eta \mathbf{e}^{T} \mathbf{D}^{T} \mathbf{V}^{T}\left(\mathbf{e}_{\mathbf{u}}-\mathbf{e}\right) .
$$

This can be seen by noting that, by a straightforward computation, the $t^{\text {th }}$ element of De is $e_{t}-\gamma e_{t+1}$, and the $t^{\text {th }}$ element of $\mathbf{V}^{T}\left(\mathbf{e}_{\mathbf{u}}-\mathbf{e}\right)$ is

$$
\sum_{k=1}^{t}(\gamma \lambda)^{t-k}\left(e_{\mathbf{u}, k}-e_{k}\right)
$$

We also used the identity $(\mathbf{D e})^{T}-\mathbf{e}^{T} \mathbf{D}^{T}$

Using the fact that $2 \mathbf{p}^{T} \mathbf{q}<\left\|\left.\mathbf{p}\right|^{2}+\right\| \mathbf{q} \|^{2}$ for any pair of vectors $\mathbf{p}, \mathbf{q} \in \mathbb{R}^{\ell}$, we can upper bound Eiq. $(8)$, for any $b>0$, by

$$
\eta^{2} X_{\lambda}{ }^{2} \mathbf{e}^{T} \mathbf{D}^{T} \mathbf{D e}-2 \eta \mathbf{e}^{T} \mathbf{D}^{T} \mathbf{V}^{T} \mathbf{e}+\frac{\eta^{2}}{h} \mathbf{e}^{T} \mathbf{D}^{T} \mathbf{V}^{T} \mathbf{V D e}+b \mathbf{e}_{\mathbf{u}}^{T} \mathbf{e}_{\mathrm{u}}
$$

(where we use $\mathbf{p}=(\eta / \sqrt{b})$ VDe and $\left.\mathbf{q}-\sqrt{b} \mathbf{e}_{\mathbf{u}}\right)$. Defining

$$
\mathbf{M}=\eta^{2} X_{\lambda}{ }^{2} \mathbf{D}^{T} \mathbf{D} \quad \eta\left(\mathbf{V D}+\mathbf{D}^{T} \mathbf{V}^{T}\right) ! \frac{\eta^{2}}{b} \mathbf{D}^{T} \mathbf{V}^{T} \mathbf{V D},
$$

and noting that $e^{T} \mathbf{D}^{T} \mathbf{V}^{T} e-e^{T} \mathrm{VDe}$, we can write Eq. (9) simply as

$$
\mathrm{e}^{T} \mathrm{Me}+b \mathrm{e}_{\mathrm{u}}{ }^{\top} \mathrm{e}_{\mathrm{a}} .
$$

Note that $\mathbf{M}$ is symmetric. It is known that in this case

$$
\max _{\mathrm{e} \neq 0} \frac{\mathrm{e}^{f} \mathrm{Me}}{\mathrm{e}^{T} \mathrm{e}}=\rho(\mathbf{M})
$$

where $\rho(\mathbf{M})$ is the largest eigenvalue of $\mathbf{M}$. (See, for instance, Horn and Johnson (1985) for background on matrix theory.) Thus, for all vectors $\mathbf{c}, \mathbf{c}^{T} \mathbf{M c}_{\mathrm{c}}<\rho(\mathrm{M}) \mathrm{e}^{T} \mathrm{e}$. It follows from $\mathrm{Eq}$. (7) that

$$
-\left\|\mathbf{w}_{1} \quad \mathbf{u}\right\|^{2} \leq b \sum_{t=1}^{t} e_{\mathbf{u}} t^{2}+\rho(\mathbf{M}) \sum_{t=1}^{t} e_{t}{ }^{2}
$$

so 


$$
\cdots \rho(\mathbf{M}) \sum_{t=1}^{\ell} e_{t}{ }^{2} \leq\left\|\mathbf{w}_{1}-\mathbf{u}\right\|^{2}+b \sum_{t=1}^{\ell} e_{\mathbf{u}, t^{2}} .
$$

In the appendix, we complete the proof by arguing that $\rho(\mathbf{M})<\cdots C_{b}$.

Having proved Lemma 1 in gory generality, we are now ready to apply it to some special cases to obtain bounds that are far more palatable. We begin by considering the case that $\lambda-0$. Note that $\operatorname{TD}(0)$ and $\operatorname{TD}^{*}(0)$ are identical.

THEOREM 1 let $0<\gamma<1$, and let $S$ be any sequence of instances/reinforcements. Assume that we know a bound $X_{0}$ for which $\left\|\mathbf{x}_{t}\right\| \leq X_{0}$.

If $\mathrm{TD}^{*}(0)$ uses any stat vector $\mathbf{w}_{1}$ and learning rates $\eta t \cdots \cdots \cdots 1 /\left(X_{1)^{2}}{ }^{2}+1\right)$, we have for all $\ell>0$ and for all $\mathbf{u} \in \mathbb{R}^{N}$.

$$
L^{\ell}\left(\mathrm{TD}^{*}(0), S\right) \leq \frac{\left(1+X_{0}{ }^{2}\right)\left(L^{\ell}(\mathbf{u}, S)+\left\|\mathbf{w}_{1}-\mathbf{u}\right\|^{2}\right)}{1-\gamma^{2}} .
$$

Assume further that we know hounds $K$ and $I /$ surh that for some nu we hove $r r^{k}(1, S) \leq$ $K$ and || $\mathbf{w}_{1}-\mathbf{u}|| \leq U$. Then for the learning rate

$$
\eta \quad \eta \cdot \frac{U}{X_{0} \sqrt{K}+X_{0}{ }^{2} U}
$$

we have that

$$
L^{\ell}\left(\mathrm{TD}^{*}(0), S\right) \leq \frac{L^{\ell}(\mathbf{u}, S): 2 U X_{0} \sqrt{K}+X_{0}{ }^{2}\left\|\mathbf{w}_{1}-\mathbf{u}\right\|^{2}}{1-\gamma^{2}} .
$$

Proof: When $\lambda \ldots 0, C_{b}$ simplifics to

$$
2 \eta \quad \eta^{2}\left(X_{0}{ }^{2}+\frac{1}{b}\right)\left(1+\gamma^{2}\right)-2 \gamma\left|\eta-\eta^{2}\left(X_{0}{ }^{2}+\frac{1}{b}\right)\right| .
$$

To minimize the loss bound given in Lemma 1, we need to maximize $C_{b}$, with respect to $\eta$. It can be shown that, in this case, $C_{b}$ is maximized, for fixed $b$, when

$$
\eta-\frac{1}{X_{0}{ }^{2}+1 / 6}
$$

The first bound (Eq. (11)) is then obtained by choosing $b=1$.

If bounds $K$ and $V$ are known as stated in the theorem, an optimal choice for $b$ can be derived by plugging the choice for $\eta$ given in Fq. (13) into the bound in Iemma 1, and replacing $L^{\ell}(\mathbf{u}, S)$ by $K$ and $\left\|\mathbf{u}-\mathbf{w}_{1}\right\|^{2}$ by $U^{2}$. This gives

$$
\frac{\left(b K-V^{2}\right)}{1-\gamma^{2}}
$$


which is minimized when $b=U /\left(X_{0} \sqrt{K}\right)$. Plugging this choice of $b$ into the bound of Lemma 1 (and setting $\eta$ as in Eq. (13)) gives the bound

$$
\begin{aligned}
& \frac{\left(U L^{\ell}(\mathbf{u}, S) / X_{0} \sqrt{K}+\left\|\mathbf{u}-\mathbf{w}_{1}\right\|^{2}\right)\left(X_{0}{ }^{2}+X_{0} \sqrt{K} / U\right)}{1-\gamma^{2}} \\
& =\frac{L^{\ell}(\mathbf{u}, S)+L^{\ell}(\mathbf{u}, S) X_{0} U / \sqrt{K}+\left\|\mathbf{u}-\mathbf{w}_{1}\right\|^{2} X_{0} \sqrt{K} / U+\left\|\mathbf{u}-\mathbf{w}_{1}\right\|^{2} X_{0}{ }^{2}}{1-\gamma^{2}} \\
& \leq \frac{L^{\ell}(\mathbf{u}, S)+2 X_{0} U \sqrt{K}+\| \mathbf{u}-\mathbf{w}_{1} !^{2} X_{0}{ }^{2}}{1-\gamma^{2}} .
\end{aligned}
$$

Next, we consider the case that $\lambda=1$.

'L'HEOREM 2 Let $0 \leq \gamma<1, \ell>0$ and let $S$ be any sequence of instances/reinforcements. Assume that we know a bound $X_{1}$ for which $: \mathbf{X}_{t}^{1}|| \leq X_{1}$, and that we know bounds $K$ and $U$ such that for some $\mathbf{u}$ we have $L^{\ell}\left(\mathbf{u}_{y} S\right) \leq K$ and $\left\|\mathbf{w}_{\mathbf{1}} \cdot \mathbf{u}\right\| \leq U$. Then if $\mathrm{TD}^{+}(1)$ uses any start vector $\mathrm{w}_{1}$ and learning rates

$$
\eta_{t}=\eta=\frac{U}{U X_{1}^{2}(1+\gamma)^{2}+X_{1}(1+\gamma) \sqrt{K}}
$$

then

$$
L^{\ell}\left(\operatorname{TD}^{*}(1), S\right) \leq L^{\ell}(\mathbf{u}, S)+2 \sqrt{K}(1+\gamma) U X_{1}+(1+\gamma)^{2}\left\|\mathbf{w}_{1} \quad \mathbf{u}\right\|^{2} X_{1}{ }^{2} .
$$

Proof: When $\lambda-1$.

$$
C_{b}-2 \eta-\eta^{2} X_{1}^{2}(1+\eta)^{2} \cdot \frac{\eta^{2}}{b}
$$

This is maximized, with respect to $\eta$, when

$$
\eta_{7} \cdots X_{1}^{2}(1+\gamma)^{2}+1 / b
$$

Proceding as in Theorem 1, we see that the best choice for $b$ is

$$
b-\frac{U}{(1+\gamma) X_{1} \sqrt{K}} .
$$

Plugging into the bound in Lemma 1 completes the theorem.

The bound in Eq. (2) is obtained from Theorem 2 by setting $w_{1} \ldots 0$. and noting that

$$
|| \mathbf{X}_{i}^{\prime} \mid<\sum_{k=1}^{t} \gamma^{t} k^{k}\left\|\mathbf{x}_{k}\right\|<\frac{\max \left\{|| \mathbf{x}_{k} \mid: 1 \leq k \leq t\right\}}{1-\gamma}
$$



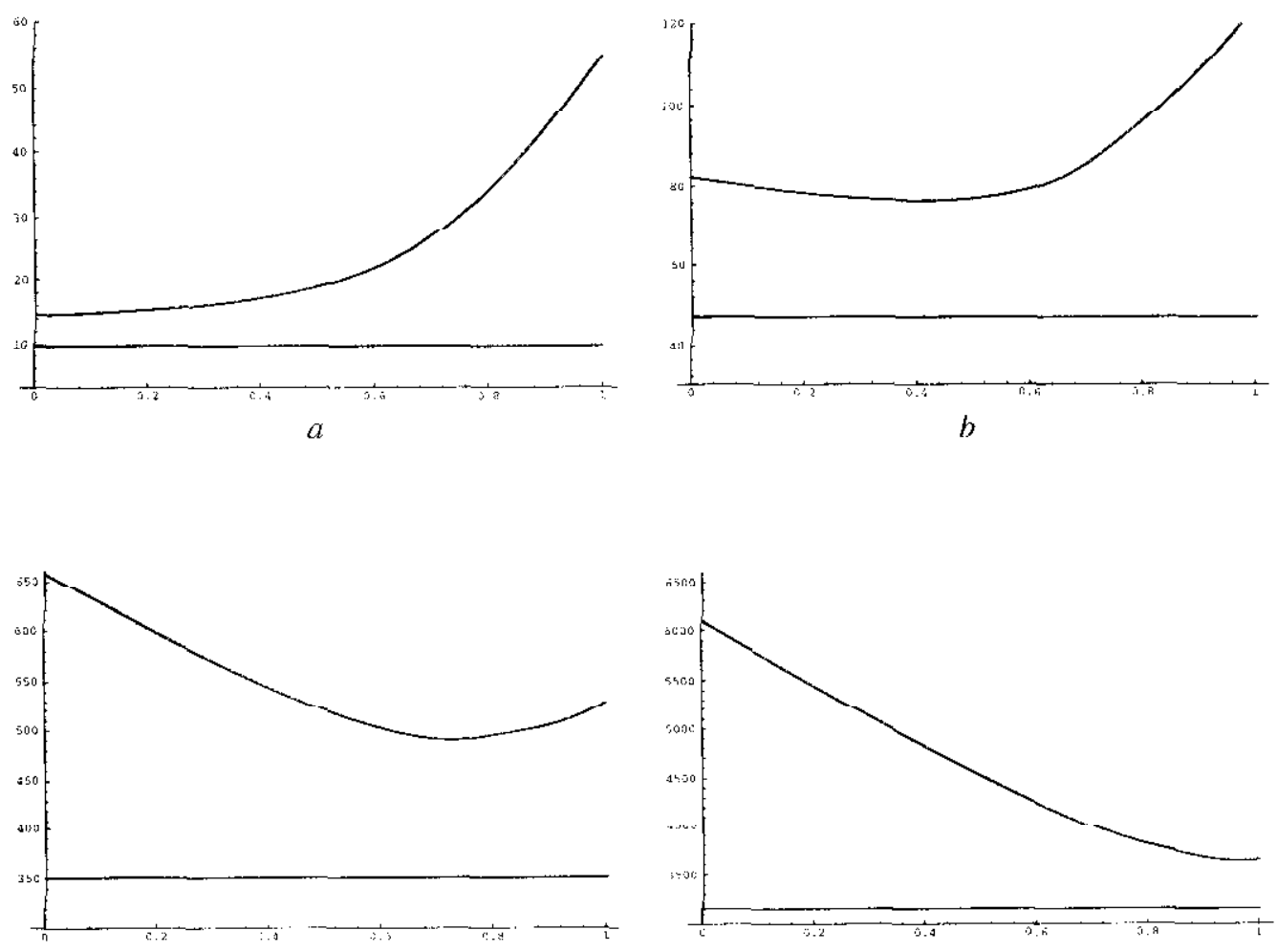

$d$

Figure ? The loss bound given in lemma $l$ as a function of $\lambda$ when $n$ is chosen so as to minimize the bound

by the triangle inequality; thus, $X_{1}$ can be replaced by $X_{0} /(1-\gamma)$. Note that the bounds in Eqs. (2) and (12) are incomparable in the sense that, depending on the values of the quantities involved. either bound can be better than the other. This suggests that TD* $(0)$ may or may nor be better than $\mathrm{T}^{\mathrm{T}} \mathrm{D}^{*}(1)$ depending on the particular problem at hand; the bounds we have derived quantify those situations in which each will perform better than the other.

Ultimately, we hope to extend our analysis to facilitate the optimal choice of $\eta>0$ and $\lambda \in[0,1]$. In the meantime, we can numerically find the choices of $\eta$ and $\lambda$ that minimize the worst case bound given in L Lemma 1. Fig. 2 shows graphs of the worstcase bound given in Iemma 1 as a function of $\lambda$ when $\eta$ is chosen so as to minimize our worst-case bound and for tixed settings of the other parameters. More specilically, in all the graphs we have assumed $\left.|| w_{1} \quad x\right|_{;}-=1$, and $\left|x_{t}\right|<1$ (which implics that $\left|i \mathbf{X}_{i}^{\lambda}\right|<1 /(\mathrm{l} \cdots \gamma \lambda)$ ). We have also fixed $\gamma-0.7$. Figs. $2 \mathrm{a}, \mathrm{b}, \mathrm{c}$ and $\mathrm{d}$ assume that $L^{\ell}(\boldsymbol{u}, S\rangle$ equals $3,30,300$ and 3000 , respectively, and each curve shows the upper bound on $L^{f}\left(\mathrm{TD}^{*}(\lambda) . s^{\prime}\right)$ given in Lomma 1 . The straight solid line in cach figure shows 
the lower bound obtained in Section 5. In each figure the $x$-axis crosses the $y$-axis at the value of $L^{\ell}(\mathbf{u}, S)$. Note that the gap between the lower bound and $L^{\ell}(\mathbf{u}, S)$ grows as $O\left(\sqrt{L^{f}(u, S)}\right)$ when all other variables are kept constant. (This is not visible fronl the figures because the scaling of the figures varies.) The figures were produced using Mathematica.

As the figures clealy indicate, the highes the loss $L^{\ell}(u, S)$, the higher should be our choice for $\lambda$. It is interesting that in some intermediate cases, an intermediate value for $\lambda$ in $(0,1)$ is the best choicc.

\section{A lower bound}

We next prove a lower bound on the performance of any learning algorithm in the model that we have been considering.

'I.HEOREM 3 Let $\gamma \in\left[0,1, X_{0}>0, K>0, U>0\right.$ and $\ell$ a positive integer For every algorithm $A$, there exists a sequence $S$ such that the following hold:

1|| $\mathbf{x}_{t} \| \leq X_{0}$.

2. $K-\min \left\{L^{\ell}(\mathbf{u}, S):\|\mathbf{u}\|<U\right\}$, and

3. $L^{\ell}(A, S)>\left(\sqrt{K}, U X_{0} \sqrt{\sigma_{\ell}}\right)^{2}$

where $\sigma_{\ell}:-\sum_{k=0}^{\ell} 1 \gamma^{2 k}$.

Proof: The main idea of the proot is to construct a thaining sequence in which the learning algorithm $A$ receives essentially no information until trial $\ell$, at which time the adversary can force the learner to incur significant loss relative to the best lincar predictor.

Without loss of genciality, we prove the result in the one-dinterisinal case ${ }^{4}$ (i.e., $N-1)$, so we write the instance $x_{t}$ simply as $x_{t}$. The sequence $S$ is defined as follows: We let $x_{t}-\gamma^{\ell}{ }^{t} X_{0}$ for $t \leq \ell$, and $x_{t}=0$ for $t>\ell$ (thus satisfying part 1 ). The reinforcement given is $r t \quad 0$ if $\ell \neq \ell$, and $r \ell$. s $z$ where $<-V X_{0}-\sqrt{K / \theta_{\ell}}$ and $s \in\{-1,+1\}$ is chosen adversarially after $A$ has made predictions $\hat{y}_{1}, \ldots, \hat{y}_{\ell}$ on the first $\ell$ trials. Then

$$
y_{t}-\sum_{k=0}^{\infty} \gamma^{k} r_{t+k}- \begin{cases}\gamma^{t-t} s z & \text { if } t \leq \ell \\ 0 & \text { otherwisc. }\end{cases}
$$

To see that part 2 holds, let $\mathbf{u}-u$ be any vector (scalar, really, since $N-1$ ) with $|u| \leq \ell$. Then

$$
\begin{aligned}
I^{\ell}(u, S)- & \sum_{t=1}^{\ell}\left(u x_{t}+y_{t}\right)^{2} \\
& -\sum_{t=1}^{k} \gamma^{2(\ell \cdots r)\left(u X_{0}-s z\right)^{2}} \\
\cdots & \left(u X_{0}-\ldots, z\right)^{2} \sigma_{t} .
\end{aligned}
$$


Since $|u|<U$, it can be seen that this is minimized when $u-s U$, in which case $L^{\ell}(u, S)=K$ by $z^{\prime}$ s definition.

Finally, consider the loss of $A$ on this sequence:

$$
L^{\ell}(A, S)=\sum_{t=1}^{\ell}\left(\hat{y}_{t} \cdot y_{t}\right)^{2}-\sum_{t=1}^{\ell}\left(\hat{y}_{t}-s \gamma^{\ell \cdot t} z\right)^{2} .
$$

For any real numbers $p$ and $q$, we have $(p-q)^{2}+(p+q)^{2}=2\left(p^{2}+q^{2}\right) \geq 2 q^{2}$. Thus, if $s \in\{-1,+1\}$ is chosen uniformly at random, then $A$ 's expected loss will be

$$
\begin{aligned}
& \frac{1}{2}\left(\sum_{t=1}^{\ell}\left(\hat{y}_{t}-\gamma^{\ell-t} z\right)^{2}: \sum_{t=1}^{\ell}\left(\hat{y}_{t}+\gamma^{\ell} t z\right)^{2}\right) \\
& \geq \sum_{t=1}^{\ell}\left(\gamma^{\ell-t} z\right)^{2}=z^{2} \sigma_{\ell}=\left(\sqrt{K}+U X_{0} \sqrt{\sigma_{\ell}}\right)^{2} .
\end{aligned}
$$

It follows that for the choice of $s \in\{-1,+1\}$ that maximizes $A$ 's loss, we will have that $L^{\ell}(A, S) \geq\left(\sqrt{K}+U X_{0} \sqrt{\sigma_{\ell}}\right)^{2}$ as claimed.

When $K=0$, Theorem 3 gives a lower bound of $U^{2} X_{0}{ }^{2} / \sigma_{\ell}$ which approaches $U^{2} X_{0}{ }^{2} /\left(1-\gamma^{2}\right)$ as $\ell$ becomes large. This lower bound matches the second bound of Theorem 1 in the corresponding casc. Thus, in the "noisc-frec" case that there cxists a vector $\mathbf{u}$ that perfectly matches the data (i.e., $\min \left\{L^{\ell}(\mathbf{u}, S):\|\mathbf{u}\| \leq U\right\}-0$ ), this shows that $\mathrm{TD}^{*}(0)$ is "optimal" in the sense that its worst-case performance is best possible.

\section{An application to Markov processes}

For the purposes of illustration, we show in this section how our results can be applied in the Markov-process setting more commonly used for studying temporal-difference algorithms. Specifically, we prove a kind of convergence theorem for $\operatorname{TD}^{*}(1)$.

We consider Markov processes consisting of a finite set of states denoted $1,2, \ldots, N$ An agent moves about the Markov process in the usual manner: An initial state $i_{1}$ is chosen stochastically. Then, at each time step $t$, the agent moves stochaslically from state $i_{t}$ to state $i_{t-1}$ where $i_{t+1}$ may depend only on the preceding state $i_{t}$. Upon exiting state $i_{t}$, the agent receives a probabilistic reward $r_{f}$ which also may depend only on $i_{t}$.

Formally, the Markov process is defined by a transition matrix $\mathbf{Q} \subset[0,1]^{N \times N}$ and an initial state distribution matrix $\mathrm{p}_{\perp} \in[0,1]^{N}$. The entries of each column of $\mathbf{Q}$ sum to 1 , as do the entries of $\mathbf{p}_{1}$. The interpretation here is that the initial state $i_{1}$ is distributed according to $\mathbf{p}_{1}$, and, if the agent is in state $i_{t}$ at time $t$, then the next state $i_{t+1}$ is distributed according to the $i_{t}^{\text {th }}$ column of $\mathbf{Q}$. Thus, state $i_{t}$ has distribution $\mathbf{p}_{t}=\mathbf{Q}^{t \cdots 1} \mathbf{p}_{l}$

The reward $r_{t}$ received at time $t$ depends only on the current state $i_{i}$ so formally we can write $r_{t}-r\left(\omega_{t}, i_{i}\right)$ where $\omega_{1}, \omega_{2}, \ldots$ are independent identically distributed random variables from some event space $\Omega$ and $r: \Omega \times\{1, \ldots N\} \rightarrow \mathbb{R}$ is some fixed function 
Let $V_{i}$ denote the expected discounted reward for a random walk produced by the Markov process that begins in state $i$. That is, we define the value function

$$
V_{i}:=\mathrm{E}\left[\sum_{k=0}^{\infty} \gamma^{k} r_{1+k}: i_{1}-i\right]
$$

where, as usual, $\gamma \in[0,1)$ is a fixed parameter. Our goal is to estimate $V_{i}$, a problem often referred to as valuc-function approximation.

At each time step $t$, the learner computes an estimate $\hat{V}_{2}^{t}$ of the value function. Thus, learning proceeds as follows. $\Lambda$ t time $t=1,2, \ldots, \ell$ :

1. The learner formulates an estimated valuc function $\hat{V}_{i}^{t}$.

2. The current state $i_{t}$ is observed.

3. The current reward $r_{t}$ is observed.

4. The learner moves to the next state $i_{t+1}$.

The states $i_{t}$ and rewards $r_{t}$ are random variables defined by the stochastic process described above. All expectations in this section are with respect to this random process.

Theorem 4, the main result of this section, gives a bound for ${ }^{T} D^{*}(1)$ on the average expected squared distance of $\hat{v}_{i}^{t}$ to the correct values $V_{i}^{t}$. Specifically, we show that

$$
\frac{1}{\ell} \sum_{l=1}^{\ell} \mathrm{F}\left[\left(\hat{V}_{i_{\ell}}^{t}-V_{i_{t}}\right)^{2}\right] \leq O\left(\frac{1}{\sqrt{ } \ell}\right)
$$

for some setting of the learning rate, and given certain benign assumptions about the distribution of rewards. Note that $\mathrm{E}\left[\left(\hat{V}_{i_{t}}^{t}-V_{i_{t}}\right)^{2}\right]$ is the expected squared distance between the $t^{\text {th }}$ estimate $\hat{V}_{i}^{t}$ and the true value function $V_{i}$ where the expectation is with respect to the stochastic choice of the $t^{\text {th }}$ state $i_{t}$. Thus, the states more likely to be visited at step t receive the greatest weight under this expectation. Theorem 4 states that the average of these expectations (over the first $\ell$ lime steps) rapidly drops to zero.

We apply $\mathrm{TD}^{*}(1)$ to this problem in the most natural manner. We define the observation vector $\mathbf{x}_{t} \in \mathbb{R}^{N}$ to have a 1 in component $i_{t}$, and 0 in all other components. (The generalization to other state representations is straightforward.) We then execute $\operatorname{TD}^{*}(1)$ using the sequence $\mathbf{x}_{1}: r_{1}, \mathbf{x}_{2}, r_{2}, \ldots, \mathbf{x}_{\ell}, r_{\ell}$ where these are tandom variables defined by the Markov process.

The estimated value function $\hat{V}_{i}^{t}$ is computed as follows: Recall that $\mathrm{TD}^{*}(1)$, at each time step $t$, gencrates an estimate $\hat{y}_{t}$ of the discounted sum $y_{t}=\sum_{k=0}^{\infty} \gamma^{k} r_{t+k}$. Note that if we are in state $i$ at time $t$, then the expected value of $y_{t}$ is exactly $V_{i}$, i.e.,

$$
\mathrm{E}:\left[y_{i}: i_{i}=i\right]-V_{i} \text {. }
$$

So it makes sense to use the estimate $\hat{y}_{t}$ in computing the $t^{\text {th }}$ value-function approximation $\hat{V}_{i}^{l}$. 
A minor difficulty arises from the fact that $\hat{y}_{t}$ is computed after $\mathbf{x}_{t}$ is observed (and therefore after $i_{t}$ is observed), but $\hat{V}_{i}^{l}$ must be computed for all states $i$ before $i_{t}$ is observed. However, if we fix the history prior to trial $t$, then $\hat{y}_{t}$ is a function only of $\mathbf{x}_{t}$, which in turn is determined by $i_{t}$. Therefore, for cach state $i$, we can precompute what the value of $\hat{y}_{t}$ will be if $i_{t}$ turns out to be $i$. We then define $\hat{V}_{i}^{t}$ to be this value. Note that, with this definition, the estimate $y_{t}$ computed by $\mathrm{TD}^{-1}(1)$ once $t_{t}$ is observed is equal to $\hat{V}_{i_{t}}^{t}$.

We now state and prove our convergence theorem. For this result, we assume a finite upper bound both on the value function and on the varlance of the sum of discounted rewards.

THEOREM 4 Suppose the Markov process is such that, for all $i,\left|V_{i}\right| \leq V$ and

$$
\mathrm{E}\left[\left(\sum_{k=0}^{\infty} \gamma^{k} r_{1+k}-V_{i}^{r}\right)^{2} \quad i_{1}=i\right] \leq R
$$

for finitc and known $V$ and $R$.

Suppose that $\mathrm{TD}^{*}(1)$ is executed as described above with $\mathbf{w}_{1}-\mathbf{0}$ and learning rate

$$
\eta_{t}=\eta-\frac{V \sqrt{N}}{J^{2}+J \sqrt{R \ell}}
$$

where $J-V \sqrt{N}(1+\gamma) /(1-\gamma)$. Then

$$
\frac{1}{\ell} \sum_{t=1}^{\ell} \mathrm{F}\left[\left(\hat{V}_{i_{t}}^{t}-V_{i_{2}}\right)^{2}\right] \leq 2 \gamma \sqrt{\frac{R}{\ell}}+\frac{J^{2}}{\ell}
$$

Proof: From Eq. (14), $\left\|\mathbf{X}_{t}^{1}\right\|<1 /(1 \quad \gamma)$ so we choose $X_{1}=1 /\left(\begin{array}{ll}1 & \gamma\end{array}\right)$. Let $\mathbf{u}$ be such that $u_{i}=V_{i}$. Then $\mid \mathbf{u} \|<U$ where $U=V \sqrt{N}$. Finally, let $K=R \ell$. Our choice for $\eta$ is identical to that in Theorem 2 where the appropriate substitutions have been madc.

Note that

$$
\mathrm{E}\left[\left(y_{t}-V_{i_{k}}\right)^{2}\right]-\sum_{i=1}^{N} \operatorname{Pr} i_{i}-i_{-} \cdot \mathrm{E}\left[\left(\begin{array}{ll}
\sum_{k=0}^{\infty} \gamma^{k} r_{t+k} & V_{i}
\end{array}\right)^{2} \mid i_{t}-i\right] \leq R
$$

by the assumption in Eq. (15). Thus, because $\mathbf{u} \cdot \mathbf{x}_{t}-V_{i}$,

$$
\mathrm{E}\left[L^{\ell}(\mathbf{u}, S)\right]-\sum_{t=1}^{\ell} \mathrm{E}\left[\left(\mathbf{u} \cdot \mathbf{x}_{t}-y_{t}\right)^{2}\right]-\sum_{t=1}^{\ell} \mathrm{E}\left[\left(V_{i_{l}} \cdot y_{t}\right)^{2}\right] \leq R \ell=K .
$$

Takiug expectations of botle sides of the bound in I emma 1, we have that 


$$
\mathrm{E}\left[L^{\ell}\left(\mathrm{TD}^{*}(\lambda), S\right)\right] \leq \frac{b \mathrm{E}\left[L^{\ell}(\mathbf{u}, S)\right]+\left\|\mathbf{u}-\mathbf{w}_{1}\right\|^{2}}{C_{b}}
$$

for any $b>0$ for which $C_{b}>0$. Therefore, by a proof identical to the proof of Theorem 2 (except that we assume only that $L^{\ell}(u, S)$ is bounded by $K$ in expectation), we have

$$
\mathrm{E}\left[I^{\ell}\left(\mathrm{TD}^{*}(1), S\right)\right] \leq \mathrm{E}\left[L^{\ell}\left(\mathbf{u}, S^{\prime}\right)\right]+2 \sqrt{K}(1+\gamma) U X_{1}+(1+\gamma)^{2}\|\mathbf{u}\|^{2} X_{1}^{2} \cdot(16)
$$

Since $\mathbf{u} \cdot \mathbf{x}_{t}=V_{i_{t}}, \hat{y}_{t}=\hat{V}_{i_{t}}^{t}$, and $\mathrm{E}\left[y_{t} \mid i_{t}\right]-V_{i_{t}}$, it can be verificd that

$$
\left.\mathrm{E}\left(y_{t}-y_{t}\right)^{2}-\left(y_{t} \cdot \mathbf{u} \cdot \mathbf{x}_{t}\right)^{2}\right]-\mathrm{E}\left[\left(V_{i_{t}}-\hat{V}_{i_{t}}^{t}\right)^{2}\right] .
$$

The theorem now follows by averaging over all time steps $t$ and combining with Eq. (16).

Unfortunately, we do not know how to prove a convergence result similar to Theorem 4 for $\operatorname{TD}^{*}(\lambda)$ for general $\lambda$. This is because this prool technique requires a worst-case bound in which the term $L^{\ell}\left(\mathbf{u}, S^{\prime}\right)$ appears with a coefficicnt of 1.

Of course, Theorem 4 represents a considerable weakening of the worst-case results presented in Section 4. These worst-case bounds are stronger because (1) they are in terms of the actual discounted sum of rewards rather than its expectation, and (2) they do not depend on any statistical assumptions. Indeed, the generality of the results in Section 4 allows us to say something meaningful about the behavior of $1 \mathrm{D}^{*}(\lambda)$ for many similar but more difficult situations such as when

- there ale a very large or cven an infinite number of states (a state can be any vector in $\mathbb{R}^{N}$ ).

- some states are ambiguously represented so that two or more states are represented by the same vector.

- the underlying transition probabilitics are allowed to change with time.

- each transition is chosen entirely or in part by an adversary (as might be the case in a game-playing scenario).

\section{Algorithm for the batch model}

In the usual supervised learning setting, the on-line learning procecds as follows: In each trial $t \geq 1$ the learncr receives an instance $x_{f} \subset{ }^{N}$. Then, after producing a prediction $\hat{y}_{t}$ it gets a rcinforcement $y_{t}$ and incurs loss $\left(y_{t} \cdots y_{t}\right)^{2}$.

A classical algorithm for this problem is the Widrow-Hoff algorithm. It keeps a linear hypothesis represented by the vector $\mathbf{w}_{t}$ and predicts with $\hat{y}_{t}-\mathbf{w}_{t} \cdot \mathbf{x}_{t}$. The weight vector is uplated using gradient descint. 
$\mathbf{w}_{t+1}:==\mathbf{w}_{t}-2 \eta_{t}\left(\mathbf{w}_{t} \cdot \mathbf{x}_{t}-y_{t}\right) \mathbf{x}_{t}$

Note that $2\left(\mathbf{w}_{t} \cdot \mathbf{x}_{t}-y_{t}\right) \mathbf{x}_{t}$ is the gradient of the loss $\left(\mathbf{w}_{t} \cdot \mathbf{x}_{t}-y_{t}\right)^{2}$ with respect to $\mathbf{w}_{t}$

There is a straightforward generalization of the above scenario when more than one instance is received in each trial $t$. In this generalization, the learner does the following in each trial:

1. receives a real-valued matrix $\mathbf{M}_{t}$ with $N$ columns;

2. computes a prediction $\hat{\mathbf{y}}_{t}$;

3. gets reinforcement $\mathbf{y}_{t}$; both $\mathbf{y}_{t}$ and $\hat{\mathbf{y}}_{t}$ are real column vectors whose dimension is equal to the number of rows of $\mathbf{M}_{t}$;

4. incurs loss $\left\|\hat{\mathbf{y}}_{t}-\mathbf{y}_{t}\right\|^{2}$.

The rows of the matrix $\mathbf{M}_{t}$ can be viewed as a batch of instances received at trial $t$. The algorithm has to predict on all instances received in trial $t$ before it gets the reinforcement vector $y_{t}$ which contains one reinforcement per row. For each instance, the algorithm is charged for the usual square loss, and the loss in trial $t$ is summed over all instances received in that trial.

The algorithm we study, called WHM, is a direct generalization of the Widrow-Hoff algorithm and was previously analyzed in the noise-frec case by Cesa-Bianchi, Long and Warmuth (1993), The learner maintains a weight vector $\mathrm{w}_{t} \in \mathbb{R}^{N}$ and, on cach trial $t$, computes its prediction as

$$
\hat{\mathbf{y}}_{t}:: \mathbf{M}_{i} \mathbf{w}_{i}
$$

After receiving reinforement $y_{t}$, the weight vector is updated using the rule

$$
\mathbf{w}_{t+1}:-\mathbf{w}_{t}-2 \eta_{t} \mathbf{M}_{t}^{T}\left(\mathbf{M}_{t} \mathbf{w}_{t}-\mathbf{y}_{t}\right)
$$

Note that the Widrow-Hoff algorithm is a special case of WHM in which each matrix $\mathbf{M}_{t}$ contains exactly one row. Also, the update is standard gradient descent in that $2 \mathbf{M}_{t}^{T}\left(\mathbf{M}_{t} \mathbf{w}_{t}-\mathbf{y}_{t}\right)$ is the gradient of the lose $\mid \mathbf{M}_{t} \mathbf{w}_{t}-\mathbf{y}_{t} \|^{2}$ with respect to $\mathbf{w}_{t}$

To model a parcicular reinforcement learning problem, we have the freedom to make up the matrices $\mathbf{M}_{t}$ and reinforcements $\mathbf{y}_{t}$ to suit our purpose. For example, Sutton (1988) and others have considered a model in which the learner rakes a random walk on a Markov chain until it reaches a terminal state, whereupon it receives some feedback, and starts over with a new walk. The learner's goal is to predict the final outcome of each walk. This problem is really a special case of nur modet in which wo let $M_{t}$ contain the instances of a run and set $y_{t}-\left(z_{t}, \cdots, z_{t}\right)^{T}$. where $z_{t}$ is the reinforcement received for the $t^{\text {th }}$ run. (In this case, Sulton shows that the Widrow-Hoff algorithm is actually equivalent to a version of $T D(1)$ in which updates are not made to the weight vector $w_{t}$ until the final outcome is received.)

An example is a pair $\left(\mathbf{M}_{t}, \mathbf{y}_{t}\right)$, and, as before, we use $S$ to denote a sequence of examples. Wo write $L^{\ell}(A, S)$ to denote the total loss of algorithm $A$ on sequence $S$ : 


$$
L^{\ell}(A, S):-\sum_{t-1}^{\ell}\left(\hat{\mathbf{y}}_{t}-y_{t}\right)^{2},
$$

where $\hat{y}_{l}$ is the prediction of $A$ in the $t^{\text {th }}$ trial, and $\ell$ is the total length of the sequence. Similarly, the total loss of a weight vector $\mathbf{u} \in \mathbb{K}^{N}$ is defined as

$$
L^{\ell}\left(\mathbf{u}, S^{\prime}\right):=\sum_{t=1}^{\ell}\left(\mathbf{M}_{t} \mathbf{u}-\mathbf{y}_{t}\right)^{2} .
$$

The proof of the following lemma and theorem are a straightforward generalization of the worst-case analysis of the Widrow-Hoff algorithm given by Cesa-Bianchi, I ong and Wannul, (1993). In the proof, we define, $|\mathbf{M}|$, the norm of any matrix $\mathbf{M}$, as

$$
\left\|\mathbf{M} \mid ! \max _{\| \mathbf{x}||=1}\right\| \mathbf{M x} \| .
$$

For comparison to the results in the first part of this paper, it is useful to note that $\|\mathbf{M}\| \leq X \sqrt{m}$ where $m$ is the number of rows of $\mathbf{M}$, and $X$ is an upper bound on the norm of each row of $M$

For any vector $\mathbf{x}$, we write $\mathrm{x}^{2}$ to denote $\mathrm{x}^{T} \mathbf{x}$.

LEMMA 2 Let $(\mathbf{M}, \mathbf{y})$ be an arbitrary example such that $\|\mathbf{M}\| \leq M$. Let $\mathrm{s}$ and $\mathrm{u}$ be any weight vectors. Let $b>0$, and let the learning rate be

$$
\eta \cdot \frac{1}{2\left(: \mathbf{M}||^{2}+1 / 6\right)} .
$$

Then

$$
\left|\mathbf{M s}-\mathbf{y}\left\|^{2}<\left(M^{2} b+1\right) \mid \mathbf{M u}-\mathbf{v}\right\|^{2}+\left(M^{2} ; 1 / b\right)\left(\|\mathbf{u}-\mathbf{s}\|^{2} \quad \cdot \mathbf{u} \cdots \mathbf{w} \|^{2}\right),\right.
$$

where $\mathbf{w}-s-2 \eta \mathrm{M}^{T}(\mathrm{Ms}-\mathrm{y})$ denotes the weight vector of the algorithm $\mathrm{WHM}$ after updating its weight vector s.

Proof: Let e : $-\mathbf{y}-\mathbf{M s}$ and $\boldsymbol{e}_{\mathbf{x}}:=\mathbf{y} \ldots \mathbf{M u}$. Then inequality (17) holds if

$$
f:-j u-w w^{2}-\mathfrak{u} \mathbf{s} \|^{2}+2 \eta \mathbf{e}^{2}-b \mathbf{e}^{2}<0 \text {. }
$$

Since $w-s+2 \eta \mathrm{M}^{T} \mathrm{e}, f$ can be rewritten as

$$
\begin{aligned}
& f \quad-\quad-4 \eta(\mathbf{u}-\mathbf{s})^{T} \mathbf{M}^{\top} \mathbf{e}+4 \eta^{2}\left|\mathbf{M}^{\top} \mathbf{e}\right|^{2}+2 \eta \mathbf{e}^{2}-b \mathbf{e}_{\mathbf{u}}{ }^{2} \\
& --4 \eta\left(\mathrm{e}-\mathbf{e}_{\mathbf{u}}\right)^{x} \mathrm{e}+4 \eta^{2}: \mathbf{M}^{f} \mathbf{e} \|^{2}: 2 \eta \mathrm{c}^{2}-b \mathrm{e}_{\mathbf{u}}{ }^{2} \\
& -2 \eta \mathrm{e}^{2}-4 \eta \mathrm{e}_{\mathrm{u}}{ }^{2} \mathrm{e}+4 \eta^{2}\left|\mathbf{M}^{T} \mathrm{e}^{2}\right|^{2}-6 \mathrm{e}_{\mathrm{u}}{ }^{2} \text {. }
\end{aligned}
$$

Since $2 \mathbf{e}_{11}{ }^{T} \mathrm{e} \leq \frac{b}{2 \eta} \mathbf{e}_{\mathrm{t}}{ }^{2}+\frac{2 \eta}{b} \mathbf{e}^{2}$ and since $\left\|\mathbf{M}^{\prime} \mathbf{e}_{\mid} \leq\right\| \mathbf{M}\|\| \mathbf{e}_{i} \mid$, we can upper bound $f$ by

$$
\mathrm{e}^{2}\left(2 \eta-4 \eta^{2}\left(\mathbf{M} \|^{2}, 1 / b\right)\right)-0
$$


THEOREM 5 Let $S$ be any sequence of examples and let $M$ be the largest norm $\left\|\mathbf{M}_{t}\right\|$. If the matrix algorithm WHM uses any start vector s and learning rates

$$
\eta_{t}=\eta=\frac{1}{2\left(\left\|\mathbf{M}_{t}\right\|^{2}+M^{2}\right)},
$$

then we have for any vector $\mathbf{u}$ the bound

$$
L^{\ell}(\mathrm{WHM}, S)<2\left(L^{\ell}(\mathbf{u}, S)+M^{2}\|\mathbf{s}-\mathbf{u}\|^{2}\right) .
$$

Assume further that we know bounds $K$ and $U$ such that for some $\mathbf{u}$ we have $L^{\ell}(\mathbf{u}, S)<$ $K$ and $\|\mathbf{s} \quad \mathbf{u}\|<U$. Then for the learning rates

$$
\eta_{t}=\eta=\frac{U}{2\left(\mid ! \mathbf{M}_{t} \|^{2} U+M \sqrt{K}\right)}
$$

we have

$$
L^{\ell}\left(\mathrm{WHM}_{,}, S\right)<L^{\ell}(\mathbf{u}, S)+2 M U \sqrt{K}+M^{2}\|\mathbf{s} \quad \mathbf{u}\|^{2} .
$$

Proof: Assume that

$$
\eta_{t}=\eta=\frac{1}{2\left(\|\mathbf{M}\|^{2}+1 / b\right)}
$$

for some $b>0$ to be chosen later. By summing the inequality of Lemma 2 over all runs of $S$ we get

$$
L^{\ell}\left(\mathbf{W H M}, s^{\prime}\right)<\left(b M^{2}+1\right) L^{\ell}\left(\mathbf{u}, S^{\prime}\right)+\left(M^{2}, 1 / b\right)\left(\mid \begin{array}{llll}
\mathbf{u} & \mathbf{s} \|^{2} & \| \mathbf{u} & \left.\mathbf{w}^{\prime} \|^{2}\right),
\end{array}\right.
$$

where $\mathrm{w}^{\prime}$ is the weight vector after the last reinforcement of $S$ is processed. Since || $\mathbf{u}-\left.\mathbf{w}^{\prime}\right|^{2}=0$, we have

$$
L^{\ell}(\mathrm{WHM} S)<\left(b M^{2}+1\right) L^{\ell}\left(\mathbf{u}, S^{\prime}\right)+\left(M^{2}+1 / b\right) \cdot \mid \mathbf{u}-\mathbf{s} \|^{2} .
$$

Now setting $b-1 / M^{2}$ gives the cholce of $\eta$ in the first part of the theorem and so yields the bound in Fq. (18).

Assuming further that $L^{\ell}(\mathbf{u}, S)<K$ and $|\mathbf{s} \quad \mathbf{u}| \mid \leq U$, we get

$$
L^{\ell}(\text { WHM }, S) \leq L^{\ell}(\mathbf{u}, S)+M^{2} \mid \mathbf{s}-\mathbf{u} \|^{2}+b K M^{2}+U^{2} / b .
$$

The part of the right hand side that depends on $b$ is $b \mathrm{KM}^{2}: V^{2} / b$ which is minimized when $b-\zeta /(M \sqrt{K})$. Using this value of $b$ in $\mathrm{Eq}$. (20) gives the desired choice of $\eta$ and the bound in Eq. (19).

In the special case that $K=0$, setting $\eta_{m}=1 /\left(2 i \mathbf{M}_{t} i^{2}\right)$ grives a bound of

$$
r \ell(W H M, S) \leq r^{\ell}\left(\mathbf{n}, S^{\prime}\right)+M^{2}: \mid \mathbf{s}-\mathbf{n} \|^{2}
$$


Note that to prove this, $b=\infty$ is used. The bound for $K=0$ was previously proved by Cesa-Bianchi, Long and Warmuth (1993). An alternate proof of the above theorem via a reduction from the corresponding theorcm for the original Widrow-Hfoff algorithm was recently provided by Kivinen and Warmuth (1994).

The following lower bound shows that the bounds of the above theorem are best pussible.

T'meorem 6 Let $N, m \geq 1, K, U \geq 0$ and $M>0$. For every prediction algorithm $A$ there exists a sequence $S$ consisting of a single example (M, $y$ ) such that the following hold:

1. $\mathbf{M}$ is an $m \times N$ matrix and || $\mathbf{M}||-M$ :

2. $K-\min \left\{I^{\ell}(\mathbf{u}, S):|| \mathbf{u} \mid \leq U\right\}$; and

3. $L^{H}(A, S) \geq K+2 U M \sqrt{K}+U^{2} M^{2}$.

Proof: As in the proof of Theorem 3, we prove the result in the case that $N-1$, without loss of generality. Thus, $M$ is actually a column veclor in $T R^{m}$.

Let each component of $\mathbf{M}$ be equal to $M / \sqrt{m}$ so that $: \mathbf{M}||-M$. I et each component of $y$ be equal to $s z$ where $z=(M U+\sqrt{K}) / \sqrt{m}$ and $s<\{1+1\}$ is chosen adversariatly after $A$ has made its prediction $\mathrm{y} \ldots\left(\hat{y_{1}}, \ldots, \hat{y}_{m}\right)^{T}$.

To sec that part 2 holds, let $\mathbf{u}-u$ be a vector (scalar, really). Then

$$
L^{\ell}(u, S)-\|\mathbf{M u} \quad \mathbf{y}\|^{2}-m(M u / \sqrt{m} \quad s z)^{2}
$$

which is minimized when $u \ldots s U$ for $|u| \leq U$. In this case, $L^{k}(u, S)=K$.

Finally, by choosing $s$ adversarially to maximize algorithm $A$ 's loss. we have

$$
\begin{aligned}
L^{k}(A, S) & -\max _{s \in\{-1,+1\}} \sum_{i=1}^{m_{2}}\left(\hat{y}_{i} s z\right)^{2} \\
& \geq \frac{1}{2} \sum_{i=1}^{m_{2}}\left(\left(\hat{y}_{i}-z\right)^{2}+\left(\hat{y}_{i}+z\right)^{2}\right) \\
& >\sum_{i=1}^{m} z^{2} K+2 M U \sqrt{K}-M^{2} U^{2}
\end{aligned}
$$

\section{Discussion}

The primary contribution of this paper is the analysis of some simple temprial-difference algorithms using a worst-case approach. This method of analysis differs dramatically from the statistical approach that has been used in the past for such problems, and ou approach has : :ome important advantages. 
First, the results that are obtained using the worst-case approach are quitc robust. Obviously, any analysis of any learning algorithm is valid only when the assumed conditions actually hold in the real world. By making the most minimal of assumptions - and, in particular, by making no assumptions at all about the stochastic nature of the work - we hope to be able to provide analyses that are as robust and broadly applicable as possible.

Statistical methods for analyring on-line learning algorithms are only neccssary when worst-case bounds cannot be obtained. In this paper, we demonstrated that temporaldifference learning algorithms with simple linear models; are highly amenable to worst case analysis. Although one might expect such a pessimistic approach to give rather weak results, we have found, somewhat surprisingly, that very strong bounds can often be proved even in the worst ease.

Worst-case bounds for on-line linear learning algorithms can be very tight even on artificial data (Kivinen \& Warmuth, 1994). Good experimental performance of a particular algorithm might be seen as weak evidence for showing that the algorithm is good since every algorithm performs well on some data, particularly when the data is artificial. However, if we have a worst-case bound for a particular algorithm, then we can use experimental data to show how much worse the competitors can perform relative to the worst-case bound of the algorithm in question.

Another strength of the worst-case approach is its emphasis on the actual performance of the learning algorithm on the actually obierved data. Breaking with more traditional approaches, we do not analyze how well the learning algorithm performs in expectation. or how well it performs asymptotically as the amount of training data becomes infinite, or how well the algorithm estimates the underlying parameters of some assumed stochastic model. Rather, we focus on the quality of the learner's predictions as measured against the finite sequence of data that it actually observes.

Finally, our method of analysis seems to be more fine grained than previous approaches. As a result, the worst-case approach may help to resolve a number of open issues in temporal-difference learning, such as the following:

- Which learning rules are best for which problems" We use the total worst-case loss as our criterion. Minimizing this criterion led us to discover the modified learning rule $\operatorname{TD}^{*}(\lambda)$. Unlike the original $\operatorname{TD}(\lambda)$. this rule has a gradient descent interpretation for general $\lambda$. Our method can also be used to derive worst-case bounds for the original rule, but we were unable to obtain bounds for $\mathrm{TD}(\lambda)$ stronger than those given for $\mathrm{TD}^{*}(\lambda)$. It will be curious to see how the two rules compare experimentally.

Also, the results in Section 4 provide explicit worst-case bounds on the performance of $\mathrm{TT}^{*}(0)$ and $\mathrm{TD}^{*}(1)$. These bounds show that one of the two algorithms may or may not be tetter than the other depending on the values of the parameters $X_{0}$, $K$, ete. Thus, using a priori knowledge we may have about a particular learning problem, we cars use these bounds to guide as in deciding which algorithm to use.

- How should a learning algorithm's parameters be tuned? For instance, we have shown how the learning rate $\eta$ should be chosen for TD" $(0)$ and $\mathrm{TD}^{*}(1)$ using knnwledge which may he available ahout a particular problem. For the choice of $\lambda$. 
Sutton showed experimentally that, in some cases, the learner's hypothesis got closest to the target when $\lambda$ is chosen in $(0,1)$ and that there is clearly one optimal choice. So far, our worst-case bounds for $\operatorname{TD}^{*}(\lambda)$ are not in clused fon when $\lambda \in(0,1)$, but, numcrically, we have found that our results are entirely consistent with Sutton"s in this regard.

- How does the performance of a learning algorithm depend on various parameters of the problem? For instance, our bounds show explicitly how the performance of $\operatorname{TD}^{*}(\lambda)$ degrades as $\gamma$ approaches 1 . Furthermore, the lower bounds that can sometimes be proved (such as in Section 5) help us to understand what performance is best possible as a function of thesc parameters.

Open problems. There remain many open research problems in this arca. The first of these is to reduce the bound given in Lemma 1 to closed form to facilitate the optimal choice of $\lambda \in[0,1]$. IIowever, as clcarly indicated by Fig. 2, even when $\lambda$ and $\eta$ are chosen so as to minimize this bound, there remains a significant gap between the upper bounds proved in Section 4 and the lower bound proved in Section 5. This may be a weakncss of our analysis, or this may be an indication that an algorithm better than either $\operatorname{TD}(\lambda)$ or $\operatorname{TD}^{*}(\lambda)$ is waiting to be discovered.

So far, we have only been able to obtain results when the comparison class consists of linear predictors detined by a weight vector $\mathbf{u}$ which make predictions of the form $\mathbf{u} \cdot \mathbf{x}_{t}$. It is an open problem to prove worst-case loss bounds with respect to other comparison classes.

As described in Section $3, \operatorname{TD}^{*}(\lambda)$ can be motivated using gradient descent Rules of this kind can alternatively be derived within a framcwork described by Kivinen and Warmuth (1994). Morcover, by modifying one of the parameters of their framework, they show that update rules having a qualitatively different flavor can be derived that use the approximation of the gradient $\nabla_{\mathbf{w}_{t}}\left(y_{t} \cdot \hat{y}_{t}\right)^{2}$ in the exponent of a multiplicative update. (Note that the $\operatorname{TD}(\lambda)$ update is additive.) In particular, they analyze such an algorithm, which they call $\mathrm{EG}$, for the same problem that we are considering in the special case that $\gamma-0$. Although the bounds they obtain are generally incomparable with the bounds derived for gradient-descent algorithms, these new algorithms have great advantages in some very important cases. It is straightforward to generalize their update rule for $\gamma>0$, but the analysis of the resulting update rule is an open problem (although we have made some preliminary progress in this direction).

Lastly, Sutton's TD $(\lambda)$ algorithm can be vicwed as a special case of Watkin's " $Q$ learning" algorithm (1989). This algorithm is meant to handle a setting in which the learner has a set of actions to choose from, and attempts to choose its actions so as to maximize its total payoff. A very interesting open problem is the cxiension of the worst-case approach to such a setting in which the learner has partial control over its cnvironment and over the foedback that it receives. 


\section{Acknowledgments}

We are very grateful to Rich Sutton for his continued feedback and guidance. Thanks also to Satinder Singh for thought-provoking discussions, and to the anonymous referees for their careful reading and feedback.

Manfred Warmuth acknowledges the support of NSF grant IRI 9123692 and AT\& T Bell Laboratories. This research was primarily conducted while visiting AT\&T Bell Laboratories.

\section{Appendix}

In this technical appendix, we complete the proof of Lemma 1 by bounding $\rho(\mathbf{M})$, the largest eigenvalue of the matrix $\mathbf{M}$.

Let $\mathbf{I}$ be the $\ell \times \ell$ identity matrix. and, for $i, j \geq 0$. define

$$
\begin{aligned}
\mathbf{S}_{i} & =\mathbf{Z}_{i}: \mathbf{Z}_{i}^{f^{\prime}} \\
\mathbf{R}_{i} & -\mathbf{Z}_{i}^{T} \mathbf{Z}_{i} \\
\mathbf{P}_{i j} & -\mathbf{Z}_{i}^{Y} \mathbf{Z}_{j}+\mathbf{Z}_{j}^{\prime} \mathbf{Z}_{i} .
\end{aligned}
$$

Since $\mathbf{Z}_{i}$ is the rero matrix for $i \geq \ell$, we can rewrite $\mathbf{V}$ more conveniently as

$$
\mathbf{V}-\sum_{i>0}(\gamma \lambda)^{i} \mathbf{Z}_{i}
$$

By direct but tedious computations, we have that

$$
\mathbf{D}^{\prime \prime} \mathbf{D}-\mathbf{I}-\gamma \mathbf{S}_{1}+\gamma^{2} \mathbf{R}_{1}
$$

and

$$
\mathbf{V D}=\mathbf{I}+\left(1-\frac{1}{\lambda}\right) \sum_{i>1}(\gamma \lambda)^{i} \mathbf{Z}_{i}
$$

since $Z_{i} Z_{1}-Z_{i+1}$ for $i \geq 0$. Also,

$$
\begin{aligned}
\mathbf{D}^{T} \mathbf{V}^{T} \mathbf{V D}- & \mathbf{I}+\left(1 \frac{1}{\lambda}\right)^{2}\left[\sum_{i \geq 1}(\gamma \lambda)^{2 i} \mathbf{R}_{i}+\sum_{j \geq i \geq 1}(\gamma \lambda)^{i-j} \mathbf{P}_{i j}\right] \\
& +\left(1-\frac{1}{\lambda}\right) \sum_{i \geqslant 1}(\gamma \lambda)^{i} \mathbf{S}_{i}
\end{aligned}
$$

Thus, M cant be witten as.

$$
\left(\eta^{2} X_{\lambda}^{2} 2 \eta+\frac{\eta^{2}}{b}\right) \mathbf{I} \div\left(\eta^{2} X_{\lambda}^{2} \gamma+\left(\eta \frac{\eta^{2}}{b}\right) \gamma(1 \quad \lambda)\right) \mathbf{S}_{1}+\eta^{2} X_{\lambda}^{2} \gamma^{2} \mathbf{R}_{1}
$$




$$
\begin{aligned}
& +\left(1-\frac{1}{\lambda}\right)\left(\frac{\eta^{2}}{b}-\eta\right) \sum_{i \geq 2}(\gamma \lambda)^{i} \mathbf{S}_{i} \\
& +\frac{\eta^{2}}{b}\left(1-\frac{1}{\lambda}\right)^{2}\left[\sum_{i>1}(\gamma \lambda)^{2 i} \mathbf{R}_{i}+\sum_{j>i \geq 1}(\gamma \lambda)^{i+j} \mathbf{P}_{i j}\right]
\end{aligned}
$$

It is known that $\rho(A+B) \leq \rho(A)+\rho(B)$ for real, symmetric matrices $A$ and $B$. Further, it can be shown (for instance, using Eq. (10)) that

$$
\begin{aligned}
\rho(\mathbf{I}) & =1 \\
\rho\left(\mathbf{R}_{i}\right) & <1 \\
\rho\left(+\mathbf{S}_{i}\right) & \leq 2 \\
\rho\left(\mathbf{P}_{i j}\right) & <2 .
\end{aligned}
$$

Applying these bounds gives that

$$
\begin{aligned}
& \rho(\mathbf{M}) \leq \eta^{2} X_{\lambda}{ }^{2} \quad \eta \eta+\frac{\eta^{2}}{b}+\eta\left|\left(\eta-\frac{\eta^{2}}{b}\right) \gamma(1-\lambda)-\eta^{2} X_{\lambda}{ }^{2} \gamma\right|+\eta^{2} X_{\lambda}{ }^{2} \gamma^{2} \\
& -2\left(1 \frac{1}{\lambda}\right)\left|\frac{\eta^{2}}{b}-\eta\right| \sum_{\imath \geq 2}(\gamma \lambda)^{i} \\
& +\frac{\eta^{2}}{b}\left(1-\frac{1}{\lambda}\right)^{2}\left[\sum_{i>1}(\gamma \lambda)^{2 i}+2 \sum_{j>i \geq 1}(\gamma \lambda)^{i+j}\right] \\
& =-C_{b} \text {. }
\end{aligned}
$$

\section{Notes}

1. In this paper we only use one vector norm, the $L_{2}$ norm: $\|\mathbf{u}\|-\sqrt{\sum_{i=1}^{N^{N}} u_{i}^{2}}$

2. In some versions of $\operatorname{TD}(\lambda)$, this difficulty is overcome by replacing $\hat{y}_{t+1} \therefore \mathbf{w}_{t+1} \cdot \mathbf{x}_{t+1}$ in the update rule (4) by the approximation $\mathbf{w}_{\ell} \cdot \mathbf{x}_{\ell+1}$

3. The lactor of two in front of $\eta_{t}$ can be absorbed into $\eta_{t}$.

4. If $N>1$, we can reduce to the one dimensional case by zetoing all but one of the components of $\mathbf{x}_{t}$.

\section{References}

Nicoló Cesid-Bianchi, Philip M. Long, \& Manfred K. Warmuth. (1993). Worst-case quadratic loss bounds for a generalization of the Widrow-Hoff rule. In Proceedings of the Sixth Annual ACM Conference on Commututional learning Theory, pages $429-438$.

Peter Dayan. (1992). The convergence of $T D(\lambda)$ for general $\lambda$. Machine Learning, 8(3/4):341-362.

Peter Dayan \& Terrence J. Sejnowski. (1994). TD( $\lambda$ ) converges with probability 1. Machine Learning, 14(3):2953 301 .

Roger A. Horm \& Charles R. Jotonson (19R5) Matrix Amolveis Cambridge llniversity Press. 
Tornmi Jaakkola. Michael I. Jordan \& Satinder P. Singh. (1993). On the convergence of stochastic iterative dynamic programming algorithms. Technical Report 9307, MIT Computational Cognitive Science.

Jyrki Kivinen \& Manfred K. Warmuth. (1994). Additive versus exponentiated gradient updates for learning linear functions. Technicat Report UCSC-CRL-94-16, University of California Santa Cruz, Computer Research Laboratory.

Richard S. Sutton. (1988). Learning to predict by the methods of temporal differences. Machine Learning. 3:9-44.

C. J. C. H. Watkins. (1989). Learning from delayed rewards. PhD thesis, University of Cambridge, England, 1989.

Received November 2, 1994

Accepted February 23, 1995

Hinal Manuscript September 29,1995 